\title{
Inhibition in Action-Inhibitory Components in the Behavioral Activation System
}

\author{
Stefan Sütterlin ${ }^{1}$, Stein Andersson ${ }^{2}$, Claus Vögele ${ }^{1}$ \\ ${ }^{1}$ Integrative Research Unit on Social and Individual Development (INSIDE), \\ University of Luxembourg, Luxembourg City, Luxembourg \\ ${ }^{2}$ Oslo University Hospital-Rikshospitalet, Oslo, Norway \\ E-mail: stefan.suetterlin@uni.lu \\ Received June 8, 2011; revised July 19, 2011; accepted July 26, 2011
}

\begin{abstract}
Over the past two decades, the neurobiological substrates of the reinforcement theory have been discussed in terms of a behavioral activation system (BAS) and a behavioral inhibition system (BIS). While the BAS has been conceptualized as both an activating system and an approach-related system, the empirical evidence for either approach remains inconclusive. In the current study we hypothesize that the inclusion of self-regulatory capacity contributes to a better understanding of the BAS. In a sample of 29 volunteers motor response inhibition elicited by a stop-signal task and heart rate variability (HRV) as a proxy of self-regulatory capacity were related to BAS scores (BIS/BAS scales [1]). Results show significant positive associations between inhibitory capacity and the sensitivity of the behavioral activation system, suggesting markers of self-regulation as components of the BAS.
\end{abstract}

Keywords: Behavioral Activation System, Heart Rate Variability, Stop-Signal Task, Self-Regulation

\section{Introduction}

Over the past two decades, extensive research has been conducted to investigate the reinforcement sensitivity theory $[2,3]$, its neurobiological substrates, related personality traits [4] and psychopathology [5], and physiological indicators. In the original formulation of their model, Gray and colleagues [2,3] suggested a behavioral activation system (BAS) and a behavioral inhibition system (BIS), which are typically operationalized with the BIS/BASScales [1] at self-report level. However, based on their research on the neurobiological substrates of these systems, Sutton and Davidson [6] conceptualized the behavioral approach system (BAS), which is opposed to the behavioral inhibition system (BIS). If the BAS scales indeed measure behavioral activation, independent from behavioral direction, and in the more comprehensive sense of intended alterations of spatial proximity (approach or active avoidance), then this would be contrary to purely approaching behavior. BAS scores should, therefore, be positively related to physiological indicators of efficient self-and emotion-regulation. Self- regulation describes the individual's ability to adapt behaviorally, emotionally and cognitively to constantly changing environmental demands. This includes goal-directed behavior, the ability to resist temptations, to overcome competing or prepotent action tendencies, to make elaborated decisions in order to regulate emotional, cognitive and motor responses to optimize future outcome (overview [7]). Selfregulation is conceived as a personality trait and can be objectively assessed under laboratory conditions, typically via physiological and behavioral indicators of prefrontally mediated inhibitory control mechanisms, using motor response paradigms. Motor response inhibition paradigms, such as the stop-signal task (SST), induce suppression of automatized, pre-potent motor behavior in pre-defined, infrequent and unpredictable cases; they require focused attention, stimulus discrimination, choice of the appropriate reaction and its execution. These processes can be subsumed under the broader term executive functions. In the present study it is hypothesized that performance in a motor response inhibition paradigm is positively associated with BAS scores.

Resting vagal tone has been identified as a peripheral physiological correlate of BAS scores. Early research reported a positive relationship between approach-related 
behavior and resting vagal tone [8,9], preparing the ground for later findings with Carver and White's [1] BAS-scale by researchers comparing physiological measures and BAS scores $[10,11]$. The positive relationship between vagal tone and BAS scores has been interpreted in terms of mechanisms of emotional, self-regulatory, and behavioral processes, according to the evolutionary theory proposed by Porges [12-14]. Nevertheless, vagal tone at rest can also be conceived as a measure of selfregulatory and inhibitory capacity. Executive functions and their association with regulatory competence and their corresponding neurophysiological substrates have been outlined in a model of neurovisceral integration, which is complementary to Porges' more philogenetic approach. The model of neurovisceral integration describes inhibitory cortico-cardiac interactions mediated by the vagus nerve and supported by the inhibitory transmitter $\gamma$-aminobutyric acid (GABA) [15-17]. A first aim of the present study was to replicate the reported positive association between vagal tone and BAS scores. The main aim of the present study, however, was the investigation of the role of inhibition in the organization of cognition, behavior and affect. Inhibitory processes are a crucial component of behavioral adaptation. In the present study measures of inhibitory capacity are operationalized as motor response inhibition performance (percentage of correctly inhibited motor responses), inhibitory speed (stop-signal reaction time), and heart rate variability (HRV), the latter indicating vagally mediated inhibitory cardiac control. We hypothesize that these measures of inhibitory control show a positive association with BAS scores, thus supporting the assumption of BAS resembling a behavioral activation system, which is closely linked to executive functions tapping inhibitory resources required for action planning and control.

\section{Materials and Methods}

\subsection{Participants}

Twenty-nine healthy participants (20 women, 9 men) were recruited via advertisement from the staff of the Oslo University Hospital. Age ranged from 19 to 47 years $(M=29.3, S D=6.5)$. Participants received a financial compensation for taking part in the study. Exclusion criteria were self-reports of current and previous psychiatric, neurological, or cardiovascular diagnoses, and medication affecting the central nervous or cardiovascular system. The study was approved by the Regional Ethical Committee of South-Eastern Norway and all subjects gave written informed consent to participate, in accordance with the Helsinki Declaration of 1975 (as revised in 1983).

\subsection{Material and Experimental Tasks}

Stop-signal task: The "GO" stimuli consisted of the letters "S" or "B", presented on a 19-inch computer display using E-Prime software (v2.0, Psychology Software Tools, Pittsburgh, PA, 2007). Stimuli were presented in black on white background, viewing distance from the screen was $80-90 \mathrm{~cm}$. Stimuli covered an angle of approximately $3.5^{\circ} \times 2^{\circ}$ of the visual field. "GO" stimuli were presented for $500 \mathrm{~ms}$, followed by an intertrial interval (ITI) of $1500 \mathrm{~ms}$. The total number of trials was 600 ; in 150 trials $(25 \%)$ the "GO" stimulus was followed by an acoustic signal $(1000 \mathrm{~Hz}, 500 \mathrm{~ms})$ acting as a stop signal. Stimulus onset asynchrony (SOA) between "GO" and "STOP" signal was $100 \mathrm{~ms}, 200 \mathrm{~ms}$, or $300 \mathrm{~ms}$, as determined by a performance-related staircase-tracking algorithm [18], ensuring a similar level of subjective difficulty of about $50 \%$ accuracy for all participants. Participants were instructed to press a button as fast as possible as soon as either letter appears on the screen, but to inhibit their response in those cases where the auditory stop signal occurred. Recovery breaks after 200 and 400 items provided the possibility to relax.

\subsection{Physiological Assessment}

Electrocardiographic recording: Electrocardiogram (ECG) was monitored using the Einthoven configuration with disposable electrodes attached to the non-dominant wrist and the opposite ankle. To reduce the probability of movement artifacts and ensure regular breathing cycles participants were instructed to relax and close their eyes while monitoring ensued for a period of $10 \mathrm{~min}$. ECG raw data were recorded using a Neuroscan polygraph (Neuroscan, Charlotte/NC), sampled at $512 \mathrm{~Hz}$.

\subsection{Data Reduction and Statistical Analysis}

Stop-signal task: Stop-signal reaction time (SSRT) and percentage of correctly suppressed reactions in "STOP" trials were calculated following the recommendations made by Logan (for details see: $[19,20]$ ), collapsing the rank-ordered reaction times of "GO" trials into a single distribution where the SSRT is identified on basis of the probability of a response in "STOP" trials. This process is repeated for each stop signal delay for each subject. The results are then averaged over subjects within and sometimes cross stop signal delays. Stop-signal reaction time estimates the speed of the inhibitory process in milliseconds, with lower value reflecting faster inhibitory processing.

Vagal tone: offline analyses of ECG included the ex- 
traction of QRS complexes and subsequent identification of interbeat intervals (IBI) from ECG recordings. Artifacts were identified according to the recommendations from Berntson and colleagues [21] and real values estimated via interpolation of neighboring IBI using ARTiiFACT software [22]. The last $5 \mathrm{~min}$ of the $10 \mathrm{~min}$ recording session was chosen for HRV analysis in order to ensure that data reflected resting conditions. Statistical parameters of HRV $[23,24]$ were calculated using ARTiiFACT. Time domain measures included mean heart rate, RMSSD (square root of the mean squared differences of successive NN intervals) and pNN50 (the proportion derived by dividing NN50 by the total number of NN intervals (NN intervals: elapsed time between subsequent ECG-R-peaks in milliseconds). Spectral frequency measures were derived using Fast Fourier Transformation (FFT). Frequency bands were labeled as recommended by the Task Force [24] as high frequency (HF, $0.15-0.4 \mathrm{~Hz}$ ) and low frequency (LF, $0.04-0.15 \mathrm{~Hz})$ and expressed in power $\left(\mathrm{ms}^{2}\right)$ and normalized units (n.u.). Spectral frequency measures and time domain measures were used as indicators for vagal tone and thus as physiological markers of inhibitory capacity. LF/HF was interpreted as a measure for autonomic balance, whereby lower values indicate higher autonomic flexibility. All measures of vagal activity were tested for normality.

Statistical analysis: BAS subscale and total scores [1] were correlated with measures of vagal tone and motor response inhibition. Intercorrelations between measures of inhibition were calculated and tests for normality carried out to ensure that criteria for multivariate analysis applied. Where assumptions of normality were violated, non-parametric correlations were conducted. Stop-signal reaction time was tested for additionally explained variance in a stepwise multiple regression model with vagal tone entered as first predictor, SSRT as second predictor. The Statistical Package for Social Sciences (SPSS 17.0, Chicago/IL) was used for all statistical analyses.

\section{Results}

Means and standard deviations for physiological and behavioral measures of inhibition are summarized in Table 1. The HRV and SSRT measures were all in a range as previously reported in the literature [20,24-25], as was the case for BAS scales "Drive" $(M=10.04 ; S D$ $=2.15)$, "Fun Seeking" $(M=10.43 ; S D=3.35)$, "Reward Responsiveness" $(M=13.50 ; S D=4.26)$, BAS total score $(M=33.21 ; S D=9.87)$ and BIS total score $(M=$ 17.89; $S D=4.25)$ [1]. The analyses of associations between measures of vagal tone (RMSSD, pNN50, HF n.u., LF/HF) and BAS subscales "Fun seeking", "Reward responsiveness" and BAS sum scores showed significant positive associations where measures of the time domain were included (Table 2). In contrast, no such associations were found between frequency domain measures and BAS scores. Non-parametric rank-correlations between $\mathrm{HF}\left(\mathrm{ms}^{2}\right)$ and BAS scores resulted in a similar non-significant result as for the other frequency domain measures. Moderate to medium effect sizes were also found for the correlation of behavioral performance (percentage of correctly inhibited stop-trials) and BAS scores.

In a stepwise regression analysis including RMSSD and SSRT the total variance explained by the predictor RMSSD alone was $12.1 \%\left(R_{\text {adjusted }}^{2}=0.09\right), F(1,28)=3.71$, $p=0.07$. Inclusion of SSRT resulted in a $R_{\text {change }}^{2}=0.09$, $F(2,27)=3.10, p=0.09$. Stop-signal reaction time was a better predictor for BAS scores (standardized $\beta=-0.315$ ) than RMSSD in a model including both predictors $(\beta=$ 0.273 ). The resulting overall model (Figure 1) with inclusion of both predictors resulted in $21.5 \%$ of explained variance $\left(R_{\text {adjusted }}^{2}=0.15, F(2,27)=3.55, p=0.04\right)$.

\section{Discussion}

The present results are in line with previous findings on the association of vagal tone and BAS scores $[10,11]$. Time domain measures of vagal tone (RMSSD, pNN50) showed significant correlations; however, frequency domain measures of vagal tone did not reach significance. RMSSD and pNN50 have been reported to be reliable estimates of vagal activity at rest $[23,24]$. Nevertheless, the present results replicate these previous findings only partially and with reservations. The nature of the as

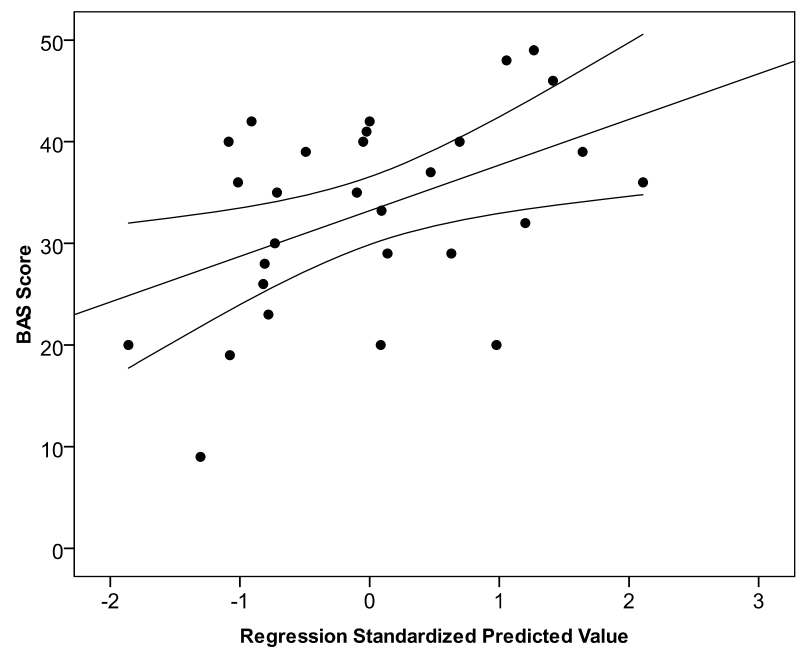

Figure 1. Scatterplot of regression model. Note: predicted and observed BAS scores in the regression model with vagal tone (RMSSD) and motor response inhibitory performance (SSRT) as predictors (curved lines represent confidence intervals to the mean). 
Table 1. Inhibitory measures.

\begin{tabular}{cccccc}
\hline & $n$ & Min & Max & Mean & $S D$ \\
\hline HRV & 29 & 19.90 & 79.00 & 39.32 & 15.65 \\
RMSSD & 29 & 1.00 & 54.20 & 20.76 & 17.12 \\
pNN50 & 29 & 58 & 555 & 217 & 150 \\
HF (ms $\left.{ }^{2}\right)$ & 29 & 17.00 & 65.70 & 44.26 & 14.97 \\
HF (n.u.) & 29 & 0.52 & 3.88 & 1.31 & 0.87 \\
LF/HF & & & & & \\
SST & 29 & 134 & 279 & 201 & 38.35 \\
SSRT (ms) & 29 & 25.79 & 94.83 & 55.77 & 18.54 \\
Correct inh. (\%) & & & & & \\
\hline
\end{tabular}

Table 2. Correlations of inhibitory measures and BIS/BAS scores.

\begin{tabular}{cccccc}
\hline & BAS Drive & BAS FS & BAS RR & BAS Sum & BIS Sum \\
\hline HRV & & & & & \\
RMSSD & 0.13 & $0.38^{*}$ & $0.35^{*}$ & $0.36^{*}$ & -0.10 \\
pNN50 & 0.13 & $0.39^{*}$ & $0.34^{*}$ & $0.37^{*}$ & -0.03 \\
HF (ms ${ }^{2}$ ) & 0.02 & 0.10 & -0.04 & 0.06 & -0.08 \\
HF (n.u.) & 0.13 & 0.02 & -0.16 & -0.07 & -0.20 \\
LF/HF & -0.09 & -0.02 & 0.17 & 0.06 & 0.17 \\
SST & & & & & \\
SSRT (ms) & -0.20 & -0.25 & $-0.37^{*}$ & $-0.39^{*}$ & -0.23 \\
Correct inh. (\%) & $0.41^{*}$ & $0.41^{*}$ & $0.38^{*}$ & 0.27 & 0.11 \\
\hline
\end{tabular}

Note: values represent Pearson's correlation coefficient $r$ for all variables with the exception of HF $\left(\mathrm{ms}^{2}\right)$. Correlations with HF $\left(\mathrm{ms}^{2}\right)$ are rank correlated (Spearman's $\left.r_{s}\right)$; * $p<0.05$. ** $p<0.01$, (one-tailed). $\mathrm{FS}=$ fun seeking, $\mathrm{RR}=$ reward responsiveness.

sumed and previously reported association between parasympathetic activation at rest and a pronounced behavioral approach or activation trait has not been specified yet, with explanations limited to, e.g., "emotional, selfregulatory, and behavioral processes" [11]. Vagal tone reflects the activity of the X. cranial nerve, mediating the cortico-cardiac modulation indicated by HRV. Neurobiological models have approached the phenomenon of respiration-induced heart-rate oscillations at rest from different perspectives. Previously, the philogenetic perspective suggested by Porges $[12,13,26]$ was referred to as an explanatory model for the observed association. In the present study a different but complementary theoretical approach was taken by deriving explicitly inhibition-oriented hypotheses from the model of neurovisceral integration [17] as a key process in the proposed central autonomic network (CAN). This network has been described as crucially depending on frontal inhibitory input and includes GABAergic neuronal networks involved in inhibitory action in emotional, cognitive, and behavioral domains $[15,17,27,28]$. The CAN depicts a model of neurovisceral integration, in which frontal inhibitory input provides the means for self-regulated action and regulated emotional responding via an extensive corticocardiac network enabling the organism to adapt flexibly to changing environmental needs, to focus attention, and to facilitate executive functioning in terms of planning and executing goal-directed behavior. Vagal activity is known to be related to inhibition-intensive processing such as working memory [29], and executive function [17] and has recently also been shown to play a role in higher-order decision-making processes such as overcoming distracting emotional biases in individual or social context [30,31]. Based on the present results it is argued that the association of vagal tone and BAS scores is linked to frontal inhibitory capacity as a component of 
executive control. This interpretation is supported by the positive relation on a behavioral level between stop-signal reaction time representing effectiveness of inhibitory processes interrupting pre-potent motor responses and BAS scores. In the light of the present findings, previous notions suggesting that vagal activity and the BAS scores are positively correlated could be revised and extended insofar as measures of inhibitory capacity are positively related to BAS scores. We concede that further research is needed to replicate these findings in larger samples, possibly applying alternative measures of inhibitory capacity such as, e.g., antisaccadic eye-movements and behavioral measures of executive control.

The role of inhibitory processes for executive functions might explain the close association with BAS scores. Executive functions and their underlying components such as goal-directed behavior, working memory, and regulated emotional responding make intensive use of prefrontally originating inhibitory processes [32]. In contrast to behavioral inhibition as indexed by the BIS-scale, the BAS occasionally requires conscious decision-making and self-regulatory competences mirrored in delay of gratification, sequential action plans and higher-order processing. Components of action control such as these are linked to prefrontal functions and inhibition in particular. They increase the likelihood of successful action and thus increase the probability of behavior as assessed by the BAS-scale.

Regarding the debate of BAS as a behavioral activation or behavioral approach system, the present findings support the idea of a behavioral activation system regardless of locomotive or motivational direction, defined as either approach or active avoidance. The concept of executive functions describes the neuronal and physiological basis for consciously planned and goal-directed behavioral competence regardless of its direction, exactly as does frontal inhibition as indexed by performance in the stop-signal task, inhibitory event-related potentials and vagal tone. Inhibitory measures constitute the organism's adaptability regardless of direction, but dependent on prefrontal neuronal activity. The present study aimed to contribute to the understanding of the mechanisms underlying the behavioral activation system with particular respect to the nature of its postulated association with vagal tone.

Recent research on relative frontal activation largely supports the concept of a behavioral activation system. In contrast to Sutton and Davidson [6], Harmon-Jones and Allen [33] reported bilateral activity to be associated with increased BAS scores. These findings were replicated by Wacker and colleagues [34], suggesting that the BAS is a behavioral activation system facilitating goaldirected behavior regardless of direction. Further con- firmation for the notion of a behavioral activation system (as opposed to a behavioral approach system) comes from a study by Hewig and colleagues [35]. In summary, these results are in line with the earlier suggestion by Gray and McNaughton [3] that active avoidance is part of the BAS. Hewig and colleagues $[35,36]$ dissected the components of motivation and affective state, and reported motivational direction to be associated with frontal asymmetry, but behavioral activation per se to be related to greater bilateral activity.

Given the controversially discussed issue regarding the operationalization of BIS/BAS and anterior asymmetry, we restricted our research to the investigation of underlying processes promoting relatively higher BASscores. Our results suggest inhibitory capacity as an endophenotypic trait marker of a pronounced behavioral activation system. We further suggest that the associations for various markers of inhibitory measures reported in the literature [10] and the results presented in the present study are in line with the assumption of a behavioral activation system, indicating higher behavioral regulation competence in individuals scoring high on the BAS scale.

Correlations of inhibitory measures and BIS score were not subject to the present study. The lack of correlations between inhibitory measures and the "behavioral inhibitory system" might appear counter-intuitive. BIS does not involve action, but the interruption and avoidance of action. High BIS scores have been reported to be associated with high reactivity to negative and potentially threatening cues and anxiety [37], the opposite of "regulated emotional responding", which has been associated with prefrontal function and vagal tone [17,27]. High BIS scores reflect poor emotion regulation. In contrast, the inhibition of behavior intuitively suggests a positive association between physiological correlates of inhibitory capacity and BIS scores, which is supported by empirical data linking dorsolateral prefrontal cortex (DLPFC) activity with BIS scores [38]. Heart rate variability is not a specific measure and involves the large multilevel model of CAN. As such it is exposed to various influences of diverging directions as they are reflected in BIS items. The BIS scale as it is conceptualized does not find an equivalent in the CAN or inhibition measures as such, particularly not a linear relationship.

\section{Conclusions}

The present results are in line with previous findings reporting a positive association of vagal tone and BAS score. This association was exceeded by a positive relationship of BAS score and motor response inhibition performance as well as the stop-signal reaction time, a 
measure of inhibitory efficacy [19]. Taken together, both measures of self-regulation and via inhibitory control complement each other in predicting BAS scores on the BIS/BAS scale. Thus, the positive association of inhibitory capacity and BAS scores provide arguments for the notion that the BAS represents a behavioral activation system, not a behavioral approach system. Inhibitory control is both, a key element of behavioral activation and executive functioning.

\section{References}

[1] C. S. Carver and T. L. White, "Behavioral Inhibition, Behavioral Activation, and Affective Responses to Impending Reward and Punishment: The BIS/BAS Scales," Journal of Personality and Social Psychology, Vol. 67, No. 2, 1994, pp. 319-333. doi:10.1037/0022-3514.67.2.319

[2] J. A. Gray, "Précis of the Neuropsychology of Anxiety: An Enquiry into the Functions of the Septo-Hippocampal System," The Behavioral and Brain Sciences, Vol. 5, No. 3, 1982, pp. 469-534. doi:10.1017/S0140525X00013066

[3] J. A. Gray and N. McNaughton, "The Neuropsychology of Anxiety: Reprise," In: D. A. Hope, Ed., Perspectives on Anxiety, Panic and Fear, University of Nebraska Press, Lincoln, 1996, pp. 61-134.

[4] J. J. Simon, S. W. Walther, C. J. Fiebach, H. C. Friederich, C. Stippich, M. Weisbrod and S. Kaiser, "Neural Processing is Modulated by Approach- and AvoidanceRelated Personality Traits," NeuroImage, Vol. 49, No. 2, 2010, pp. 1868-1874. doi:10.1016/j.neuroimage.2009.09.016

[5] M. R. M. Scholten, J. van Honk, A. Aleman and R. S. Kahn, "Behavioral Inhibition System (BIS), Behavioral Activation System (BAS) and Schizophrenia: Relationship with Psychopathology and Physiology," Journal of Psychiatric Research, Vol. 40, No. 7, 2006, pp. 638-645.

[6] S. K. Sutton and R. J. Davidson, "Prefrontal Brain Asymmetry: A Biological Substrate of the Behavioral Approach and Inhibition Systems," Psychological Science, Vol. 8, No. 3, 1997, pp. 204-210. doi:10.1111/j.1467-9280.1997.tb00413.x

[7] R. F. Baumeister and K. D. Vohs, "Handbook of SelfRegulation," The Guilford Press, New York, 2004.

[8] J. E. Richards, "Respiratory Sinus Arrhythmia Predicts Heart Rate and Visual Responses during Visual Attention in 14- and 20-Week-Old Infants," Psychophysiology, Vol. 22, No. 1, 1985, pp. 101-109. doi:10.1111/j.1469-8986.1985.tb01567.x

[9] N. A. Fox, "Psychophysiological Correlates of Emotional Reactivity during the First Year of Life," Developmental Psychology, Vol. 25, No. 3, 1989, pp. 364-372. doi:10.1037/0012-1649.25.3.364

[10] S. L. Brenner, T. P. Beauchaine and P. D. Sylvers, "A Comparison of Psychophysiological and Self-Report Measures of BAS and BIS Activation," Psychophysiology, Vol. 42, No. 1, 2005, pp. 108-115. doi:10.1111/j.1469-8986.2005.00261.x

[11] H. L. Movius and J. J. B. Allen, "Cardiac Vagal Tone, Defensiveness, and Motivational Style," Biological Psychology, Vol. 68, No. 2, 2005, pp. 147-162. doi:10.1016/j.biopsycho.2004.03.019

[12] S. W. Porges, "Vagal Tone: An Autonomic Mediator of Affect," In: J. Garber and K. A. Dodge, Eds., Principles of Psychophysiology: Physical, Social, and Inferential Elements, Cambridge University Press, Cambridge, 1991, pp. 456-512.

[13] S. W. Porges, "Orienting in a Defensive World: Mammalian Modifications of Our Evolutionary Heritage. A Polyvagal Theory," Psychophysiology, Vol. 32, No. 4, 1995, pp. 301-318. doi:10.1111/j.1469-8986.1995.tb01213.x

[14] N. Eisenberg, R. A. Fabes, B. Murphy, P. Maszk, M. Smith and M. Karbon, "The Role of Emotionality and Regulation in Children's Social Functioning: A Longitudinal Study," Child Development, Vol. 66, No. 5, 1995, pp. 1360-1384. doi:10.2307/1131652

[15] E. E. Benarroch, "The Central Autonomic Network: Functional Organization, Dysfunction, and Perspective," Mayo Clinic Proceedings, Vol. 68, No. 10, 1993, pp. 9881001.

[16] J. F. Thayer and J. F. Brosschot, "Psychosomatics and Psychopathology: Looking up and down from the Brain," Psychoneuroendocrinology, Vol. 30, No. 10, 2005, pp. 1050-1058. doi:10.1016/j.psyneuen.2005.04.014

[17] J. F. Thayer and R. D. Lane, "Claude Bernard and the Heart-Brain Connection: Further Elaboration of a Model of Neurovisceral Integration," Neuroscience and Biobehavioral Reviews, Vol. 33, No. 2, 2009, pp. 81-88. doi:10.1016/j.neubiorev.2008.08.004

[18] M. Boecker, M. M. Buecheler, M. L. Schroeter and S. Gauggel, "Prefrontal Brain Activation during Stop-Signal Response Inhibition: An Event-Related Functional NearInfrared Spectroscopy Study," Behavioural Brain Research, Vol. 176, No. 2, 2007, pp. 259-266. doi:10.1016/j.bbr.2006.10.009

[19] G. D. Logan and W. B. Cowan, "On the Ability to Inhibit thought and Action: A Theory of an Act of Control," Psychological Review, Vol. 91, No. 3, 1984, pp. 295-327. doi:10.1037/0033-295X.91.3.295

[20] G. D. Logan, "On the Ability to Inhibit Thought and Action: A User's Guide to the Stop-Signal Paradigm," In: D. Dagenbach and T. H. Carr, Eds., Inhibitory Processes in Attention, Memory, and Language, Academic Press, San Diego, 1994, pp. 184-239.

[21] G. G. Berntson, K. S. Quigley, J. F. Jang and S. T. Boysen, "An Approach to Artifact Identification: Application to Heart Period Data," Psychophysiology, Vol. 27, No. 5, 1990, pp. 586-598.

doi:10.1111/j.1469-8986.1990.tb01982.x

[22] T. Kaufmann, S. Sütterlin, S. M. Schulz and C. Vögele, "ARTiiFACT: A Tool for Heart Rate Artifact Processing and Heart Rate Variability Analysis," Behavior Research Methods, published online, 14 May 2011. doi:10.3758/s13428-011-0107-7 
[23] B. Allen, A. S. Chambers and D. N. Towers, "The Many Metrics of Cardiac Chronotropy: A Pragmatic Primer and a Brief Comparison of Metrics," Biological Psychology, Vol. 74, No. 2, 2007, pp. 243-262. doi:10.1016/j.biopsycho.2006.08.005

[24] Task Force of the European Society of Cardiology and the North American Society of Pacing and Electrophysiology, "Heart Rate Variability-Standards of Measurement, Physiological Interpretation, and Clinical Use," European Heart Journal, Vol. 17, 1996, pp. 354-381.

[25] G. J. M. van Boxtel, M. W. van der Molen, J. R. Jennings and C. H. M. Brunia, "A Psychophysiological Analysis of Inhibitory Motor Control in the Stop-Signal ParaDigm," Biological Psychology, Vol. 58, No. 3, 2001, pp. 229-262. doi:10.1016/S0301-0511(01)00117-X

[26] S. W. Porges, J. A. Doussard-Roosevelt and A. K. Maiti, "Vagal Tone and the Physiological Regulation of Emotion," In: Society for Research in Child Development, Ed., The Development of Emotion Regulation: Biological and Behavioral Considerations, Blackwell Publishing, New York, 1994, pp. 167-186.

[27] B. M. Appelhans and L. J. Luecken, "Heart Rate Variability as an Index of Regulated Emotional Responding," Review of General Psychology, Vol. 10, No. 3, 2006, pp. 229-240. doi:10.1037/1089-2680.10.3.229

[28] V. Napadow, R. Dhond, G. Conti, N. Makris, E. N. Brown and R. Barbieri, "Brain Correlates of Autonomic Modulation: Combining Heart Rate Variability with fMRI," NeuroImage, Vol. 42, No. 1, 2008, pp. 169-177. doi:10.1016/j.neuroimage.2008.04.238

[29] A. L. Hansen, B. H. Johnsen and J. F. Thayer, "Vagal Influence on Working Memory and Attention," International Journal of Psychophysiology, Vol. 48, No. 3, 2003, pp. 263-274. doi:10.1016/S0167-8760(03)00073-4

[30] S. Sütterlin, C. Herbert, M. Schmitt, A. Kübler and C. Vögele, "Frames, Decisions, and Cardiac-Autonomic Control," Social Neuroscience, Vol. 6, No. 2, 2011, pp. 169177. doi:10.1080/17470919.2010.495883

[31] S. Sütterlin, C. Herbert, M. Schmitt, A. Kübler and C. Vögele, "Overcoming Selfishness: Reciprocity, Inhibition, and Cardiac Autonomic Control in the Ultimatum Game," Frontiers in Psychology, Vol. 2, No. 173, 2011, pp. 1-8. doi: 10.3389/fpsyg.2011.0017

[32] S. Funahashi, "Neuronal Mechanisms of Executive Control by the Prefrontal Cortex," Neuroscience Research, Vol. 39, No. 2, 2001, pp. 147-165.

doi:10.1016/S0168-0102(00)00224-8

[33] E. Harmon-Jones and J. J. B. Allen, "Behavioral Activation Sensitivity and Resting Frontal EEG Asymmetry: Covariation of Putative Indicators Related to Risk of Mood Disorders," Journal of Personality and Social Psychology, Vol. 74, No. 5, 1997, pp. 1310-1316. doi: $10.1037 / 0022-3514.74 .5 .1310$

[34] J. Wacker, M. Heldmann and G. Stemmler, "Separating Emotion and Motivational Direction in Fear and Anger: Effects on Frontal Asymmetry," Emotion, Vol. 3, No. 2, 2003, pp. 167-193. doi:10.1037/1528-3542.3.2.167

[35] J. Hewig, D. Hagemann, J. Seifert, E. Naumann and D. Bartussek, "On the Selective Relation of Frontal Cortical Asymmetry and Anger-Out versus Anger-Control," Journal of Personality and Social Psychology, Vol. 87, No. 6, 2004, pp. 926-939. doi:10.1037/0022-3514.87.6.926

[36] J. Hewig, D. Hagemann, J. Seifert, E. Naumann and D. Bartussek, "The Relation of Cortical Activity and BIS/ BAS on the Trait Level," Biological Psychology, Vol. 71, No. 5, 2006, pp. 42-53. doi:10.1016/j.biopsycho.2005.01.006

[37] A. F. Jorm, H. Christensen, A. S. Henderson, P. A. Jacomb, A. E. Korten and B. Rodgers, "Using the BIS/ BAS Scales to Measure Behavioural Inhibition and Behavioural Activation: Factor Structure, Validity and Norms in a Large Community Sample," Personality and Individual Differences, Vol. 26, No. 1, 1998, pp. 49-58. doi:10.1016/S0191-8869(98)00143-3

[38] A. J. Shackman, B. W. McMenamin, J. S. Maxwell, L. L. Greischar and R. J. Davidson, "Right Dorsolateral Prefrontal Activity and Behavioral Inhibition," Psychological Science, Vol. 20, No. 12, 2009, pp. 1500-1506. doi:10.1111/j.1467-9280.2009.02476.x 